\title{
O universo masculino no domicílio: a visão dos homens acerca do Programa Melhor em Casa
}

\author{
Jocelly Ferreira de Araújo, ${ }^{1}$ Fernanda Beatriz Dantas de Freitas, ${ }^{1}$ \\ Joseane da Rocha Dantas Cavalcante, ${ }^{1}$ Jucicleia Maiara da Silva Freitas, ${ }^{1}$ \\ Alexandre Ernesto da Silva² e Wallison Pereira dos Santos ${ }^{3}$
}

Como citar Araújo JF, Freitas FBD, Cavalcante JRD, Freitas JMS, Silva AE, Santos WP. O universo masculino no domicílio: a visão dos homens acerca do Programa Melhor em Casa. Rev Panam Salud Publica. 2018;42:e123. https:/ / doi.org/10.26633/RPSP.2018.123

RESUMO Objetivo. Verificar o atendimento das necessidades de saúde na perspectiva de homens usuários do programa de atenção domiciliar Melhor em Casa.

Métodos. O presente estudo observacional e descritivo, com delineamento qualitativo, foi realizado com homens cadastrados no Programa Melhor em Casa (PMC) no município de Cuité, estado da Paraíba, Nordeste do Brasil. Foram entrevistados 10 homens dos 19 cadastrados no PMC em Cuité. Para a coleta de dados, utilizou-se entrevista norteada por um roteiro semiestruturado. Para os dados utilizou-se a análise de conteúdo de categoria temática proposta por Bardin.

Resultados. Emergiram três categorias temáticas: conhecimento dos homens acerca do problema de saúde que o condicionou à assistência domiciliar; percepção masculina quanto às necessidades de saúde apresentadas no domicílio; e percepção dos homens assistidos sobre as facilidades da assistência domiciliar. Constatou-se que os homens não conheciam aspectos importantes de sua atual condição de saúde, inclusive o motivo que os condicionou à utilização do serviço de saúde. Quanto às facilidades do serviço, foram apontadas a agilidade dos profissionais, a eliminação de grandes filas de espera características dos serviços públicos de saúde e a comodidade do ambiente domiciliar.

Conclusões. Foi notável a aceitabilidade e a satisfação com a assistência domiciliar por parte dos usuários. Entretanto, constatou-se grande desconhecimento dos entrevistados quanto às suas próprias necessidades no domicílio. Destaca-se a necessidade de novos estudos para ampliar as discussões acerca do cuidado domiciliar.

Palavras-chave Serviços de assistência domiciliar; saúde do homem; percepção; masculinidade; Brasil.

\footnotetext{
Universidade Federal de Campina Grande, Unidade Acadêmica de Enfermagem, Campus Cuité (PB), Brasil. Correspondência: Jocelly Ferreira de Araújo, jocellyaferreira@hotmail.com

2 Universidade Federal de Minas Gerais (UFMG), Programa de Pós-Graduação em Enfermagem, Belo Horizonte (MG), Brasil.

3 Universidade Federal da Paraíba, Programa de Pós-Graduação em Enfermagem, João Pessoa (PB), Brasil.
}

As políticas de saúde são definidas como resposta social do Estado diante dos problemas de saúde e dos determinantes ambientais que afetam ativamente a saúde do indivíduo e da comunidade (1). Sendo assim, como resposta aos diversos tipos de risco relevantes para a população masculina, no ano de 2009 o Ministério da Saúde do Brasil, juntamente com a comunidade científica, civil e acadêmica, instituiu a Política Nacional de Atenção Integral à Saúde do Homem (PNAISH). Essa política tem por objetivo proteger a saúde dessa população, promovendo 
ações de saúde que contribuam significativamente para a compreensão da realidade masculina nos seus diversos contextos socioculturais e político-econômicos. Em suma, a PNAISH reflete o reconhecimento de que os agravos de saúde da população masculina, a exemplo das doenças cardiovasculares, câncer de pulmão e próstata e causas externas (2), constituem verdadeiros problemas de saúde pública.

A PNAISH leva em conta o que parece ser uma resistência dos homens em adentrar o serviço de saúde pela porta da atenção primária à saúde (APS). Tal resistência causa, além de atraso no diagnóstico, um aumento considerável nos gastos com internações hospitalares no Sistema Único de Saúde (SUS). No ano de 2012, o valor médio pago por internação hospitalar no âmbito do SUS chegava a 1050,80 reais $(2,3)$.

Nesse contexto, ao refletir sobre como contornar os gastos em saúde e ao mesmo tempo atender a mudança no perfil epidemiológico que ocorre no Brasil (4) - com aumento do número de idosos e de doenças crônicas e degenerativas -, o Ministério de Saúde instituiu uma modalidade organizacional chamada atenção domiciliar (AD), que tem como um de seus eixos a desospitalização, proporcionando ao usuário um cuidado continuado no domicilio (5). Estima-se que a economia obtida com o uso de AD por usuário seja de cerca de $80 \%$ em comparação à internação hospitalar $(2,6)$.

A implantação da $\mathrm{AD}$ visou a assegurar os princípios e diretrizes do SUS, nomeadamente universalidade, integralidade, equidade, participação comunitária, descentralização, regionalização e hierarquização, e ao mesmo tempo diminuir a superlotação nos hospitais, permitindo desocupação de leitos de pacientes crônicos que pudessem receber cuidados em casa (7). Foi instituído, dessa forma, o Programa Melhor em Casa (PMC), uma modalidade inovadora de cuidado que vem contribuindo de maneira eficaz para a integralidade da assistência em saúde. O PMC é caracterizado por seu caráter substitutivo ou complementar aos demais serviços, objetivando promover, manter e restaurar a saúde do usuário (8). A implementação do PMC trouxe redução dos gastos decorrentes das longas internações hospitalares, ao mesmo tempo em que garantiu a continuidade do cuidado e a integração com as redes de atenção à saúde (9).
Considerando o perfil masculino de saúde no Brasil, associado a altos índices de doenças crônicas e degenerativas, doenças do aparelho circulatório, neoplasias e causas externas, o objetivo deste estudo foi investigar o atendimento das necessidades de saúde pelo PMC na perspectiva de homens usuários do Programa.

\section{MATERIAIS E MÉTODOS}

O presente trabalho representa um recorte do projeto "Assistência domiciliar aos homens: na perspectiva do Melhor em Casa" da Universidade Federal de Campina Grande, estado da Paraíba. Este artigo relata uma pesquisa observacional e descritiva, com delineamento qualitativo, realizada no município de Cuité, localizado no Curimataú Ocidental, região semiárida do estado da Paraíba. A pesquisa foi aprova pelo Comitê de Ética em Pesquisa do Hospital Universitário Alcides Carneiro (HUAC) (parecer 1.919.636).

No Censo de 2010, a população de Cuité era de 19978 habitantes, sendo 13132 residentes na zona urbana e 6488 residentes na zona rural. Do total, 9818 eram homens e 10132 eram mulheres (10). O estudo teve como universo populacional todos os homens cadastrados no PMC do município de Cuité. Conforme informado pelo programa, havia 19 homens cadastrados no município.

Foram considerados os seguintes critérios de inclusão: idade superior a 18 anos; ter capacidade de compreensão e verbalização; e assinar o termo de consentimento livre e esclarecido (TCLE). Quanto aos critérios de exclusão, desconsideraram-se homens cadastrados em outros programas dentro do ambiente da APS. Nove homens cadastrados no PMC não possuíam capacidade de compreensão e verbalização, uma limitação justificada pela idade avançada. Sendo assim, 10 homens foram incluídos no estudo.

Após análise ética do projeto de pesquisa, realizou-se contato prévio com a equipe do PMC para estabelecer vínculo e levantar informações acerca dos usuários cadastrados e seus endereços. Posteriormente, os pesquisadores se dirigiram aos domicílios dos homens, juntamente com a equipe de profissionais do PMC, para explicar o estudo e convidar os usuários a participarem.

Os dados foram coletados por meio de entrevista guiada por um roteiro semiestruturado, desenvolvido pelos pesquisadores, com organização fixa da ordem e da relação das questões subjetivas respondidas por todos os participantes da pesquisa. $\mathrm{O}$ discurso dos participantes foi registrado em mídia digital (tipo gravação de áudio). A coleta ocorreu entre os meses de maio e junho de 2017. Posteriormente, as entrevistas foram transcritas.

Os dados foram analisados pelo método da análise de conteúdo, proposto por Bardin (11). A análise de conteúdo desenvolve-se em três importantes fases: pré-analise; exploração do material; e tratamento, interferência e interpretação dos dados. A fim de garantir o anonimato, os participantes foram identificados pela letra " $\mathrm{H}$ " seguida de número que reflete a sequência das entrevistas.

\section{RESULTADOS}

A idade dos participantes variou de 61 até 87 anos. Cinco participantes eram casados, quatro eram viúvos e um era divorciado. Quanto à escolaridade, cinco participantes eram analfabetos e outros cinco tinham ensino fundamental incompleto.

No que diz respeito à ocupação, os participantes eram aposentados. Antes da aposentadoria, desempenhavam atividades de agricultura e comércio. No momento do estudo, estavam afastados de qualquer atividade econômica, tendo em vista a condição de saúde que levou à inclusão no PMC. Os participantes moravam com familiares, cônjuge e filhos. A renda familiar de todos os homens era proveniente unicamente da aposentadoria.

A partir dos depoimentos, foram delimitadas as unidades de codificação e as unidades de registro mais citadas, com base em palavras, frases e expressões (11). Essa análise revelou três categorias temáticas: conhecimento dos homens acerca do problema de saúde que os condicionou à assistência domiciliar (C1); percepção masculina quanto às necessidades de saúde apresentadas no domicílio (C2); e percepção dos homens assistidos sobre as facilidades da assistência domiciliar (C3). Posteriormente, os discursos foram confrontados e comparados com a literatura atual e pertinente à temática.

$\mathrm{O}$ discurso dos participantes no que diz respeito à $C 1$, ou seja, conhecimento sobre a própria condição de saúde, mostrou que alguns não conseguiam associar 
o tipo de agravo com a patologia instalada e outros caracterizavam doenças crônicas, a exemplo do diabetes melito, como uma complicação aguda, evidenciando desconhecimento da situação. Nesse sentido, foi feita a pergunta "o senhor sabe qual a doença que tem?".

Eu? Doença eu não tenho nada, tirando desse pé ai, eu não tenho nada em cima de mim [...] (H1)

[...] é melhor você perguntar a minha mulher que ela sabe de tudo, ela dizendo é mesmo que ser eu [...] (H1)

O que hoje mesmo eu sei é a diabetes, aî depois paralisou os rins, dos rins faz só 1 ano, 1 ano 5 meses mais ou menos, perdi os rins ai por isso estou fazendo hemodiálise [...] (H3)

Não tenho nada, a única doença que eu tenho na minha vida só foi essa agora [...] (H7)

Sei não, é problema de cabeça [...] (H8)

Diabetes braba e esse câncer no pescoço [...] (H9)

Não sei não, os médicos que sabem [...] (H10)

Quanto à C2, a verbalização das necessidades de saúde no domicílio revelou que alguns não consideravam ter necessidades, ou afirmavam que a necessidade era de outros, como da esposa. Outros, por sua vez, manifestaram necessidade física, social, biológica e espiritual:

Essa história de perna cortada, homem pelo amor de Deus, o cabra é de cortar as pernas de uma criatura como esse povo fizeram comigo, é melhor o cabra pegar um machado e acabar de matar caladinho [...] $(\mathrm{H} 2)$

eu me criei trabalhando [choro] e trabalhava e me dava bem nos meus trabalhos, graças a Deus [...] (H2)

Segundo os comentários das enfermeiras, evitar o máximo de beber água, por causa dos rins, porque incho muito, até essa mão aqui é inchada direto [...] E que outro dia eu estava sem defecar, sabe? Que eu passo tempão rapaz, as vezes até mês e mais de mês, entendesse? [...] (H3)

Eu tenho precisão. De melhorar. De andar, ficar vendendo as coisas [...] (H5)

Não, bom agora eu estou tendo necessidade aqui né? Mas foi um acaso, eu nunca sofri de nada, nunca tive doença [...] (H7)
Tá bom não, que ela não pode andar comigo, pra dar banho, pra dar de comer [...] (H8)

Quanto à C3, os participantes mencionaram a agilidade do programa e a ida do serviço até o domicílio, fazendo com que os participantes não precisem se locomover. Também foi mencionada a vantagem do não enfrentamento das longas filas na rede hospitalar pública.

[...] Fica mais fácil por tudo ela vindo pra cá, porque eu não posso andar [...] Aqui é bom demais porque estou em casa, não estou andando, não tem quem me leve, a mulher não pode comigo [...] (H1)

[...] A domicílio é 100\% né? Porque olhe, eu teria que me locomover, eu não tenho carro, já era uma dificuldade, como é que eu ia me locomover com a perna assim, eu sair daqui nesse anda já [...] $O$ atendimento de vocês é ótimo, não tenho do que reclamar [...] (H7)

[...] O povo fala que aqui é ruim, quem fala que aqui é ruim é porque não conhece lá fora minha filha, aqui você chega tem 4 a 10 pessoas pra ser atendido, lá fora têm é 200 [...] (H9)

[...] Em casa é melhor, sabe por quê? Porque eu não posso andar, vou daqui ali à esquina às vezes e volto porque eu não posso mais andar. Já pensou se eu fosse daqui ao posto agora? Só chegava amanhã [...] (H9)

[...] Porque pra ir para hospital é preciso um carro e eu não tenho [...] (H10)

\section{DISCUSSÃO}

No presente estudo, realizado em um município de pequeno porte no estado da Paraíba, Brasil, a maioria dos 10 homens participantes do PMC - todos eles acometidos por doenças crônicas e complicações decorrentes - verbalizou não conhecer o seu próprio problema de saúde. Embora alguns reconhecessem a existência da patologia que os condicionou à $\mathrm{AD}$, poucos assumiam a condição fragilizada de saúde e afirmavam não existir doença, mas sim um agravo temporário. Esse resultado pode estar diretamente relacionado ao nível instrução e conhecimento dos participantes. Conforme estudo anterior, esse déficit de conhecimento acerca da patologia pode levar o indivíduo a ficar ainda mais exposto a agravos que, na maior parte das vezes, podem ser evitados, dificultando também o processo de educação em saúde para esses indivíduos $(12,13)$.

Quanto aos homens que negam a situação da saúde fragilizada, esses apoiam-se nos aspectos históricos e culturais que consideram um modelo de masculinidade hegemônica, que valoriza o estereótipo de homem corajoso, viril, destemido, forte física e emocionalmente e provedor do seu lar, a exemplo dos depoimentos de $\mathrm{H} 1$ e $\mathrm{H} 7$ ao afirmaram que não tinham doença alguma.

Esse modo de entender e vivenciar a masculinidade impõe ao homem uma condição tida como não vulnerável ao adoecimento e às fragilidade sociais, conjuntura que determina uma barreira para que os homens cuidem da própria saúde, por entender que essa procura é desnecessária e por assumir essa fragilidade como uma espécie de "arranhadura" à sua masculinidade (14).

As masculinidades são referidas como práticas sociais desempenhadas pelos homens e condicionadas pelas relações de gênero. Como nas relações de gênero há interação de outros fatores, como raça, classe social e religião, é adequado falar em masculinidades no plural, uma vez que não existe um único tipo de prática social $(15,16)$. As discussões sobre masculinidades e gênero cresceram no Brasil a partir dos movimentos feministas que lutam por direitos humanos. Esse marco feminista levou a discussões a respeito das diferenças entre os sexos e do conceito biológico-sexual (14). Para alguns autores, historicamente, o conceito de gênero relacionava-se exclusivamente às mulheres, chegando até mesmo a serem utilizados como sinônimos. Contudo, com o passar do tempo, esse conceito passou a abordar também o masculino. A partir de então, estudos sobre masculinidades e gênero passaram a ter uma maior visibilidade e relevância, especialmente a partir da década de 1980 (15).

Quando questionados sobre quais necessidades apresentavam no domicílio, alguns participantes do estudo referiram não saber quais eram, enquanto outros relataram não existir nenhuma. Essa observação confirma outros achados do estudo que evidenciaram o desconhecimento dos participantes acerca da doença existente.

Uma parcela dos homens demonstrou, em suas falas, dependência de suas esposas, evidenciando a necessidade de 
cuidador, conforme H1 e H8. Foi possível observar também, no discurso dos participantes, a insatisfação pela condição de saúde em que se encontravam.

Estudos apontam que, para operacionalizar o conceito de necessidades de saúde, é preciso ratificar a dialética do individual e do coletivo. A taxonomia das necessidades de saúde criada por Cecílio (17) está dividida em quatro grupos. O primeiro é das necessidades de boas condições de vida, que engloba as necessidades fisiológicas, como alimentação, saneamento, moradia, e necessidades mais complexas, como afeto e segurança. Pode também estar relacionado com a determinação social do processo de saúde e doença.

O segundo grupo pontua a garantia de acesso a todas as tecnologias que melhorem e prolonguem a vida. $\mathrm{O}$ acesso inclui necessidades e demandas individuais, em todos os níveis de atenção, e o acesso a todas as tecnologias, podendo ser elas leves, leves-duras e duras, a depender da carência de cada usuário.

O terceiro grupo aborda a necessidade de ter vínculo com profissional ou equipe de saúde, favorecendo as práticas diárias do sujeito, possibilitando que expresse suas dificuldades, além de estimular a autonomia (18). O vínculo deve ser uma relação contínua, pessoal e intransferível, um encontro de subjetividades. Se sustenta na afetividade, aceitação e confiança, tornando-se dessa forma um elemento essencial para um atendimento satisfatório (12).

Ainda de acordo com a taxonomia de necessidades de saúde, o quarto grupo diz respeito às necessidade de autonomia e autocuidado na construção do "modo de andar a vida". Cecílio (17) afirma que as necessidades de saúde do usuário são profundamente influenciáveis por sua autonomia, uma vez que o modo como ele conduz sua vida é capaz de despertar maior interesse pela terapêutica; essa participação ativa poderá levá-lo mais rapidamente à cura, o que não é observado quando o indivíduo se apresenta como ser passivo e sem voz (17). No presente estudo, o grupo de necessidade de autonomia foi encontrado com maior frequência, manifestando-se nas expressões de desconhecimento da doença e, consequentemente, também das necessidades de saúde, com transferência da autonomia para outras pessoas, como os cuidadores.
Quanto à percepção dos homens assistidos sobre as facilidades da assistência domiciliar, pode-se inferir que todos os participantes da pesquisa destacaram como principal vantagem o fato de não precisarem se locomover até o serviço de saúde, destacando ainda a inviabilidade desse deslocamento, devido às condições de saúde fragilizadas e à falta de transporte público ou privado para a locomoção.

O deslocamento para o serviço hospitalar, somado à longa espera por atendimento, ocasiona a produção de memórias negativas. Em contrapartida, quando o serviço vai até o usuário, ele o considera como familiar e aconchegante. Observase que a $\mathrm{AD}$ tranquiliza tanto os cuidadores quanto os próprios usuários no sentido de não precisarem locomover-se até uma unidade hospitalar (18).

Os homens ainda enfatizaram a agilidade e a qualidade da AD. Um dos grandes benefícios da AD é a oferta da segurança da estrutura convencional de um hospital na comodidade do próprio domicílio (19). A AD é considerada uma excelente alternativa para ofertar cuidados especializados com comodidade e conforto, promovendo a desospitalização (20).

A agilidade do serviço também foi citada pelos participantes. Ressalta-se, contudo, que, em levantamento bibliográfico, não foram identificadas pesquisas anteriores que corroborassem esse achado. Portanto, é importante que essa observação seja confirmada novos estudos, em outras localidades e com número maior de participantes.

Quando questionados sobre as dificuldades com a $\mathrm{AD}$, a maioria dos homens afirmou não existirem dificuldades, sendo imperioso destacar a satisfação verbalizada pelos usuários em relação aos serviços ofertados. Esse achado corrobora pesquisa realizada pelo SUS por meio de ouvidoria no ano de 2012, com intuito de mensurar a satisfação dos usuários atendidos pelo PMC. Tais consultas telefônicas demonstraram um índice de satisfação de $93,9 \%$ por parte dos usuários com o PMC (21). De fato, o Ministério da Saúde já destacou a importância da realização de pesquisas periódicas a respeito da satisfação do usuário com o serviço ofertado, sendo imprescindível que esses estudos levem em consideração o contexto socioeconômico e cultural da realidade estudada (5).
Durante a realização desta pesquisa, encontraram-se algumas dificuldades, que podem ter interferido nos seus resultados finais. Uma dessas barreiras foi a mudança de gestão municipal na época de início da coleta de dados, o que ocasionou a mudança de toda equipe do PMC e o desligamento de todos os usuários cadastrados na gestão anterior. Dessa maneira, os usuários ficaram sem nenhum atendimento por algum tempo, até que acontecesse uma reorganização pela atual gestão e contratação de novos profissionais. Outro ponto importante diz respeito ao momento do convite para participação na pesquisa, em que os pesquisadores foram apresentados juntamente com a equipe de saúde. Isso pode ter levado os usuários a confundirem os pesquisadores com profissionais atuantes no PMC, caracterizando uma possível intimidação.

Foram ainda limitações do presente estudo o número pequeno de participantes com características socioeconômicas e culturais específicas, o que impede a extrapolação dos dados para outros cenários. Entretanto, foi importante identificar, no grupo estudado, a falta de reconhecimento desse grupo de suas próprias necessidades.

Em resumo, durante as entrevistas, identificou-se que uma facilidade destacada por todos os participantes foi a comodidade que a $\mathrm{AD}$ oferece, tornando desnecessária a locomoção (considerada inviável) até os serviços de saúde. No que concerne às dificuldades relativas à $\mathrm{AD}$, a maioria afirmou não existirem dificuldades que comprometessem a qualidade da assistência ofertada. Destaca-se a satisfação quase que absoluta dos usuários com os serviços. Finalmente, vale ressaltar um achado desta pesquisa não encontrado em estudos anteriores, relativo à agilidade do serviço, citada pelos usuários como uma facilidade da AD. Sendo assim, é essencial a realização de novas pesquisas para ampliar a discussão dessa temática.

Conflitos de interesse. Nada declarado pelos autores.

Declaração. As opiniões expressas no manuscrito são de responsabilidade exclusiva dos autores e não refletem necessariamente a opinião ou política da RPSP / PAJPH ou da Organização Pan-Americana da Saúde (OPAS). 


\section{REFERÊNCIAS}

1. Aguiar ZN. SUS - Sistema Único de Saúde: antecedentes, percurso, perspectivas e desafios. São Paulo: Martinari; 2015.

2. Brasil, Conselho Nacional de Medicina. Resolução CFM 1 931/2009. Disponível em: http://portal.cfm.org.br/index.php? option=com_content\&view=category\&id= 9\&Acessado em julho de 2018

3. Brasil, Ministério da Saúde. Valor médio pago por internação hospitalar no SUS (AIH). Disponível em: http:/ / tabnet.datasus.gov.br/cgi/deftohtm.exe?idb2012/ e11.def Acessado em julho de 2018.

4. Vasconcelos AMN, Gomes MMF. Transição demográfica: a experiência brasileira. Epidemiol Serv Saude. 2012;21(4):539-48.

5. Brasil, Ministério da Saúde, Departamento de Atenção Básica. Melhor em Casa: a segurança do hospital no conforto do seu lar. Caderno de Atenção Domiciliar. Vol 1. Brasília, DF: Ministério da Saúde, Secretaria de Atenção à Saúde, Departamento de Atenção Básica; 2012. Disponível em: http://189.28.128.100/dab/docs/publicacoes/geral/cad_vol1.pdf Acessado em julho de 2018.

6. Brasil, Ministério da Saúde, Fundação Oswaldo Cruz, Instituto Nacional de Saúde da Mulher, da Criança e do Adolescente Fernandes Figueira. Fortalecimento da Política Nacional de Atenção Integral à Saúde do Homem (PNAISH): compromisso versus ação na atenção básica. Brasília: Ministério da Saúde; 2013. Disponível em: http://portalarquivos.saude.gov.br/images/pdf/2014/maio/21/CNSH-DOCFortalecimento-da-PNAISH.pdf Acessado em julho de 2018.

7. Braga PP, Sena RR, Seixas CT, Castro EAB, Andrade AM, Silva YC. Oferta e demanda na atenção domiciliar em saúde. Cienc Saude Coletiva. 2016;21(3):903-12.
8. Marcolin GCA. Panorama da Atenção Domiciliar do Sistema Único de Saúde (SUS): correlatividade com os serviços de atenção primária. Teoria Soc. 2014;22(2): 254-75.

9. Rodrigues FF, Santos MA, Texeira CRS, Gonela JT, Zanetii ML. Relação entre conhecimento, atitude, escolaridade e tempo de doença em indivíduos com diabetes mellitus. Acta Paul Enferm. 2012;25(2): 284-90.

10. Instituto Brasileiro de Geografia e Estatística (IBGE). População - Cuité (PB). Brasília: IBGE; 2010. Disponível em: http://www. censo2010.ibge.gov.br/sinopse/webservice $/$ frm_urb_rur.php? codigo $=25050$. Acessado em junho de 2017.

11. Bardin L. Análise de conteúdo. Lisboa: Edições 70; 2011.

12. Brondani CM, Ramos LH, Beuter M, Lampert MA, Seiffert MA, Bruinsma. Caracterização de pacientes dependentes de tecnologias de um serviço de internação domiciliar. Rev Enferm UFSM. 2013;3(esp): 689-99.

13. Gomes MF, Turra CM, Fígoli MGB, Duarte YAO, Lebrão MC. Associação entre mortalidade e estado marital: uma análise para idosos residentes no Município de São Paulo, Brasil, Estudo SABE, 2000 e 2006. Cad Saude Publica. 2013;29(3):566-78.

14. Franco FA, Hino P, Nichiata LYI, Bertolozzi MR. A compreensão das necessidades de saúde segundo usuários de um serviço de saúde: subsídios para a enfermagem. Rev Esc Anna Nery. 2012;16(1):157-62.

15. Castro MA. Ainda somos os mesmos?: masculinidades e produção de cuidados entre duas gerações de homens Belo Horizonte [dissertação de mestrado]. Belo Horizonte: Universidade Federal de Minas Gerais; 2015. Disponível em http://www. bibliotecadigital.ufmg.br/dspace/handle/1843/ANDO-A76MMJ Acessado em junho de 2017.

16. Silva YC. A produção do cuidado na Atenção Domiciliar: relações de poder e estratégias de resistência [dissertação de mestrado]. Belo Horizonte: Universidade Federal de Minas Gerais; 2015. Disponível em: http://www.bibliotecadigital.ufmg.br/ dspace/handle/1843/ANDO-A77GZP Acessado em Junho de 2017.

17. Cecílio LCO. Apontamentos teórico-conceituais sobre processos avaliativos considerando as múltiplas dimensões da gestão do cuidado em saúde. Interface (Botucatu). 2011;15(37):589-99.

18. Connell RW, Messerschmidt JW. Masculinidade hegemônica: repensando o conceito. Rev Est Fem. 2013;21(1):241-82.

19. Oliveira SG, Quintana AM, Budo MLD, Kruse HL, Beuter M. Internação domiciliar e internação hospitalar: semelhanças e diferenças no olhar do cuidador familiar. Texto Contexto - Enferm. 2012;21(3): 591-9.

20. Katzer J, Madeira FP. Fisioterapia domiciliar em pacientes graves com acometimento respiratório. Cad Saude Desenvolvimento. 2016;8(5):64-73.

21. Neto AVO, Dias MB. Atenção domiciliar no SUS: O que representou o programa melhor em casa? Rev Divulg Saude Debate. 2014;1(51):58-71.

Manuscrito recebido 13 de novembro de 2017. Aceito em versão revisada em 28 de junho de 2018. 
ABSTRACT

The masculine universe in the home: men's views on the Better at Home Program in Brazil

Keywords Home care services; men's health; perception; masculinity; Brazil.
Objective. To investigate the perception of male users of a home care service (Programa Melhor em Casa, PMC) provided by the Unified Health System (SUS) in Brazil regarding the fulfilment of their health needs.

Method. The present observational and descriptive study, with qualitative design, was performed with men registered with PMC in Cuité, a municipality located in the state of Paraíba, Northeastern Brazil. Ten of 19 men registered with PMC in Cuité were interviewed. Data collection was based on a semi-structured interview with participants. Results were analyzed using Bardin's content analysis of thematic categories.

Results. Three thematic categories were identified: knowledge of participants regarding the health problem for which they were receiving home care; masculine perception regarding the health needs in the home; and perception of participants regarding the advantages of home care. The results showed that the men interviewed were unaware of many important aspects of their health condition, such as the reason for their inclusion in a home care program. Regarding the advantages of PMC, participants mentioned the quick response by health care workers, the avoidance of long waiting lines that are usual in public health care services, and the comfort of the home.

Conclusions. The acceptance and satisfaction of users with PMC was remarkable. However, a high level of unawareness regarding the participants' own health needs was observed. Additional studies are required to broaden the discussion regarding home care.
RESUMEN

\section{El universo masculino en el hogar: la visión de los hombres acerca del Programa Mejor en Casa}

Objetivo. Verificar la atención de las necesidades de salud desde la perspectiva de los hombres usuarios del Programa Mejor en Casa, que presta atención domiciliaria. Métodos. El presente estudio observacional y descriptivo, con delineamiento cualitativo, se realizó con hombres registrados en el Programa Mejor en Casa (PMC) en el municipio de Cuité, estado de Paraíba, nordeste de Brasil. Se entrevistaron 10 de los 19 hombres registrados en el PMC en Cuité. Los datos se recopilaron por medio de una entrevista orientada por una guía semiestructurada y se examinaron con el análisis de contenido por categoría temática propuesto por Bardin.

Resultados. Surgieron tres categorías temáticas, a saber: el conocimiento de los hombres acerca del problema de salud que dio origen a la atención domiciliaria, la percepción masculina de las necesidades de salud que se presentaron en el hogar y la percepción de los hombres que recibieron asistencia con respecto a las facilidades del servicio de atención domiciliaria. Se constató que los hombres desconocían varios aspectos importantes de su estado de salud en el momento del estudio, incluso el motivo que los llevó a utilizar el servicio de salud. En cuanto a las facilidades del servicio, se señalaron la agilidad de los profesionales, la eliminación de las largas filas de espera características de los servicios públicos de salud y la comodidad del entorno del hogar.

Conclusiones. Los usuarios demostraron un alto grado de aceptación de la atención domiciliaria y de satisfacción con ella. Sin embargo, se comprobó que desconocían en gran medida sus propias necesidades en el hogar. Se destaca la necesidad de realizar nuevos estudios para ampliar las deliberaciones sobre el cuidado domiciliario.

Palabras clave Servicios de atención de salud a domicilio; salud del hombre; percepción; masculinidad; Brasil. 